\title{
Eigen-Parameter Based Damage Detection in Sandwich Composite Ship Hull Structures
}

\author{
Carlos Toro ${ }^{\mathrm{a}}$, Basir Shafiq ${ }^{*}$, , David Serrano $^{\mathrm{a}}$, Fred Just ${ }^{\mathrm{a}}$ \\ a Department of Mechanical Engineering (P.O. Box 9045), University of Puerto Rico, \\ Mayagüez, PR 00681, USA \\ ' Department of General Engineering (P.O. Box 9044), University of Puerto Rico, \\ Mayagüez, PR 0068I, USA
}

\begin{abstract}
An eigen-parameter based technique was applied to detect localized damage in geometrically complex sandwich composite structures using 3D orthotropic finite element analysis. It was shown that the fundamental displacement mode shape curvature of a sandwich structure can be used to identify the unique damage sites corresponding to a reduction in stiffness, which show up as spikes in the curve. The technique was shown to work well for planar geometries. For non-planar or discontinuous structures, such as a ship hull, a segment wise analysis approach is suggested. This technique handled material complexities efficiently but with added mesh generation effort. A probabilistic neural network was trained and used to aid in damage detection of 3D geometries based on training from simpler cases. The results of neural network were satisfactory; however, convergence time increased with increasing structural complexity.
\end{abstract}

\section{INTRODUCTION}

Major impacts may produce audible sounds and visible damage on the surface of the composite, whereas routine minor impacts degrading the soft core or the interface between the core and the facesheets can go unnoticed. Various non-destructive techniques, such as, radiography, ultrasonics, mechanical impedance, holography, thermography, acoustic emission and vibration analysis have been applied to assess small scale damages in sandwich composites with varying degrees of success $/ 1,2 /$. However, with ever increasing geometric and material complexities in engineering applications, the search for viable damage detection techniques continues.

\footnotetext{
Corresponding Author: Phone: (787)832-4040 ext. 2094, Fax: (787)265-3816,
}

Email: b_shafiq@ige.uprm.edu 
Various vibration based experimental and finite element analysis (FEA) studies have shown that the dynamic properties (such as eigenvalues, eigenvectors and damping) change as the structure suffers damage $12,3 /$. The advantage of using eigenvalues and damping is that they can be easily measured at any point on the structure and are independent of the position chosen $/ 3-5 /$. However, excessive computational effort is required to relate changes in eigenvalues and damping to the extent and location of damage site /4-6/. Furthermore, some investigations have concluded that eigenvalues are not consistent with the location and magnitude of damage in complex structures $/ 2,7,8$; current study supports this claim.

On the other hand, it has been shown that the difference between the damaged and undamaged curvatures of eigenvectors accentuates irregularities in the curve and facilitates damage site identification with greater reliability 77 . However, it has been suggested that the accuracy of damage site location increases with the use of higher modes of vibration, a claim that is not supported by the current analysis of sandwich composites. Polynomial smoothing techniques applied to fundamental mode curvature have shown to locate damage in the laminated composites without comparison with the undamaged case $/ 8,9 /$ Results presented in this paper show that no polynomial smoothing is necessary as damage is adequately discernable from the fundamental mode displacement curvature.

The displacement curvature based approaches reported in the literature have been limited to the analysis of straight 2D beams $/ 7-9 /$. This paper extends the application of this damage detection technique to complex 3D sandwich composite and ship hull structures. The limitations of this approach are discussed and a segment-wise analysis is proposed to overcome difficulties associated with geometric irregularities. Furthermore, a probabilistic neural network (PNN) is trained using the FEA results. The neural network is used to detect location and extent of damage in the structure subjected to different scenarios than those used in the training.

\section{FINITE ELEMENT MODELING}

FEA is the most popular approach in eigenparameter based analysis as the eigenvectors and eigenvalues can be efficiently acquired for complex geometries and material compositions /4,5,7-10/. In the current study, a 3D orthotropic analysis was performed by imposing circular symmetry to the general 3D elasticity formulation, as given in the equation below,

$$
\sigma_{i j, i}+f_{j}=\rho \ddot{\Delta}_{j}
$$

where $\sigma_{\mathrm{i}, \mathrm{i}, \mathrm{i}}, \mathrm{f}_{\mathrm{j}}, \rho$ and $\ddot{\Delta}_{j}$ represent the stress, body force, mass density and generalized displacement vector, respectively. Using the stress-strain and strain-displacement relationships, differential equations were obtained to setup the weak form of displacement based orthotropic FEA /11/. Stiffness and mass matrices 
were constructed. FEA was performed to solve linear eigenvalue problem as given by the following standard cquation:

$$
\left\{[K]^{i}-\lambda[M]^{i}\right\}\left\{\Delta^{i}\right\}=0
$$

where $[\mathrm{K}]^{\mathrm{i}}$ and $[\mathrm{M}]^{\mathrm{i}}$ represent the stiffness and mass matrices, respectively. $\lambda$ represents the eigenvalues and $\Delta^{\prime}$ the associated eigenvectors. The cigenvectors were mass normalized as a standard representation.

Preliminary analysis involved optimization of mesh density for optimal solution convergence of the models at hand. Initially 2D isotropic models of various mesh densities, material types and geometries were analyzed to validate the displacement curvature method. The damage was simulated as a reduction in the local elemental stiffness. Results on material types compared favorably with the literature; however, geometric changes introduced complexities that were not encountered in the $2 \mathrm{D}$ models reported in the literature $15,7,8 /$. Furthermore, plane elasticity laminated (or sandwich composite) theory lacked in its ability to analyze damage at the interior of the composite, whereas, application of 3D orthotropic FEA provided greater flexibility in through the thickness analysis. However, 3D analysis brought greater complexity in mesh development and refinement as matching and numbering nodes consecutively on each layer, which is critical for continuous curvature calculation became a tedious process.

\section{NEURAL NETWORK}

Many different types of neural network schemes are available for pattern classification $/ 12 \%$. A combination of various neural networks has been successfully applied to identify the extent and location of damage in structural elements /12-15/. In the present study of damage detection in sandwich composites, probabilistic neural network (PNN) based on Bayes decision rule that selects the category with minimum conditional risk was implemented. The PNN offers rapid training speed, guaranteed convergence to a Bayes classifier (Bayes Optimality), robustness to noisy data and generalization ability over other neural network schemes /12/. More precisely, PNN is interpreted as a function which approximates the probability density of the underlying distribution of data (rather than directly fitting the data). Therefore, training is quick since it is done in one pass of each training vector, rather than several. In the case of minimum error rate classification, the rule selects the category with the maximum posterior probability. For example, suppose there are $c$ classes, $w_{1}, w_{2}, w_{7}, \ldots, w_{c}$. Given a feature vector $x$, the minimum crror rate rule will assign it to $w_{1}$ if

$$
P\left(w_{1} \mid x\right) \succ P\left(w_{1} \mid x\right) \text { for all i not equal to } \mathrm{j}
$$

here the posterior probability is used as the discriminant function. For every category c, a probability density 
function can be defined as

$$
f_{c}(x)=P(x \mid c)
$$

A set of training examples are used to estimate $f_{c}(x)$ for each category. Then, the Bayes (optimum) decision is applied based on these PDF's in conjunction with prior probabilities. Smooth and continuous PDF's are then approximated using PDF estimators. By following this scheme, $f_{c}(x)$ gives the sum of Gaussian distributions centered at each training pattern. In PNN, each training pattern is encoded as the input weight vector of unit $i$ called pattern unit, which leads to the appropriate design of PNN.

PNN is not designed to be an incremental learner since old examples are represented explicitly in the network. Every new example demands the modification of the network architecture by the addition of another pattern unit and associated connections. This is the main limitation of PNN. On the other hand, the learning process is computationally less complex in PNN compared to the progressively learning neural network schemes.

\section{RESULTS}

The eigenvector analysis is based on the idea that irregularities in a given function are accentuated by taking the curvature of a given function, as given by the Laplacian equation below,

$$
\text { curvature }=f^{\prime \prime}(x)=\frac{f(x+h)-2 f(x)+f(x-h)}{h^{2}}
$$

where $\mathrm{h}$ is the element width. For a modal displacement plot, such irregularities have been found to be the result of existing damage in the structure. Thus the damage site can be located by relating spikes in the otherwise smooth displacement curvature plot to the physical location of the corresponding data point. This implies that in order to locate the damage site, the node numbering must be readily known, especially on more complicated models where internal nodes corresponding to the different laminas or sandwich core material are considered. This, however, becomes a disadvantage for the proposed technique, thus necessitating development of special algorithm for consecutive node numbering or deciphering of node numbers for curvature calculation, as was performed in the current analysis.

Applying Eq. 5 to the displacement mode shape data, the damage information can be ascertained from the damaged only state. However, the curvature difference between damaged and undamaged state was observed to eliminate the false peaks that sometimes manifest as a result of certain boundary conditions and/or due to proximity of multiple damage sites, etc. Furthermore, preliminary analysis clearly indicated that analysis of higher modes of vibration did not bring additional information or clarity in damage identification and that the 
fundamental displacement mode shape curvature pertained sufficient features necessary for damage identification 17,8\%. Therefore, the results presented in the subsequent discussion are of normalized fundamental displacement mode shape curvature difference between damaged and undamaged structural element.

The two normalized peaks in Fig. 1 point to two different damage sites located at nodes 8 and 25, respectively of the isotropic model of Fig. 1 (inset). It can be seen that the damage was successfully located and that the peaks increased in magnitude as the amount of damage increased, which agrees well with the reported results $17 /$. If characterized in an accurate manner this behavior can give an idea of the amount of damage the structure has undergone. Thus the sensitivity of the technique becomes an important issue when considering small changes in stiffness. From Fig. 1, it can be seen that the curvature difference plot is very distinct for each damage location; this anomaly was the basis for using PNN as detailed later.

Both laminated and sandwich composites were analyzed using 3D orthotropic elasticity to detect facesheet, core and multiple site damage; the results compared favorably to the exact solution based on plane clasticity theory /11/. Fig. 2a presents the successful detection of a damage site (in the XY-plane) located at node 406 of row 4 within a four lamina $\left[0^{\circ}, 90^{\circ}\right]$ composite shown in Fig. 2a (inset). Relative magnitudes of damage can be observed in descending order in the neighboring rows. The results appear to be quite reasonable as the damage in an element is expected to affect the surrounding structure. However, without $a$ priori knowledge of node numbering, Fig. 2a (XY-plane analysis) does not entail through the thickness (layer-wise) location.

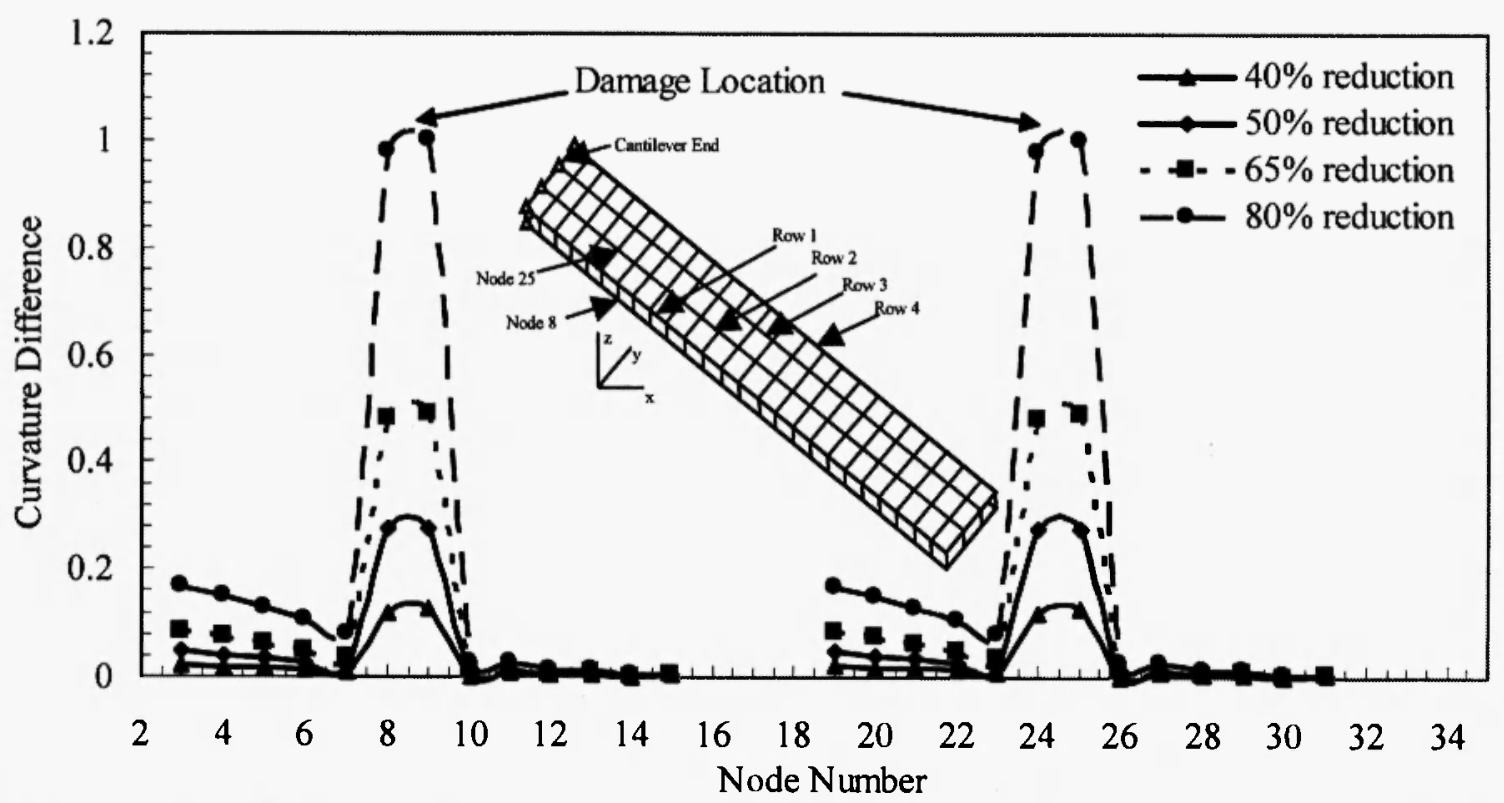

Fig. 1: Extent and location of damage relating to percentage of induced stiffness reduction at various nodes of the isotropic beam model. 
A problem arises in through-the-thickness analysis as the gradient of magnitude of out-of-plane deformation (as a result of damage in an element) is generally very small in the $\mathrm{XZ}$ plane. For example, if a beam undergoes flexure, all through-the-thickness points below any element on the surface must undergo almost similar magnitude of bending. As a result, the curvature differences in the $\mathrm{XZ}$ plane among individual layers of comparable stiffness characteristics, as given in Fig. $2 b$, show little variation in the magnitudes of

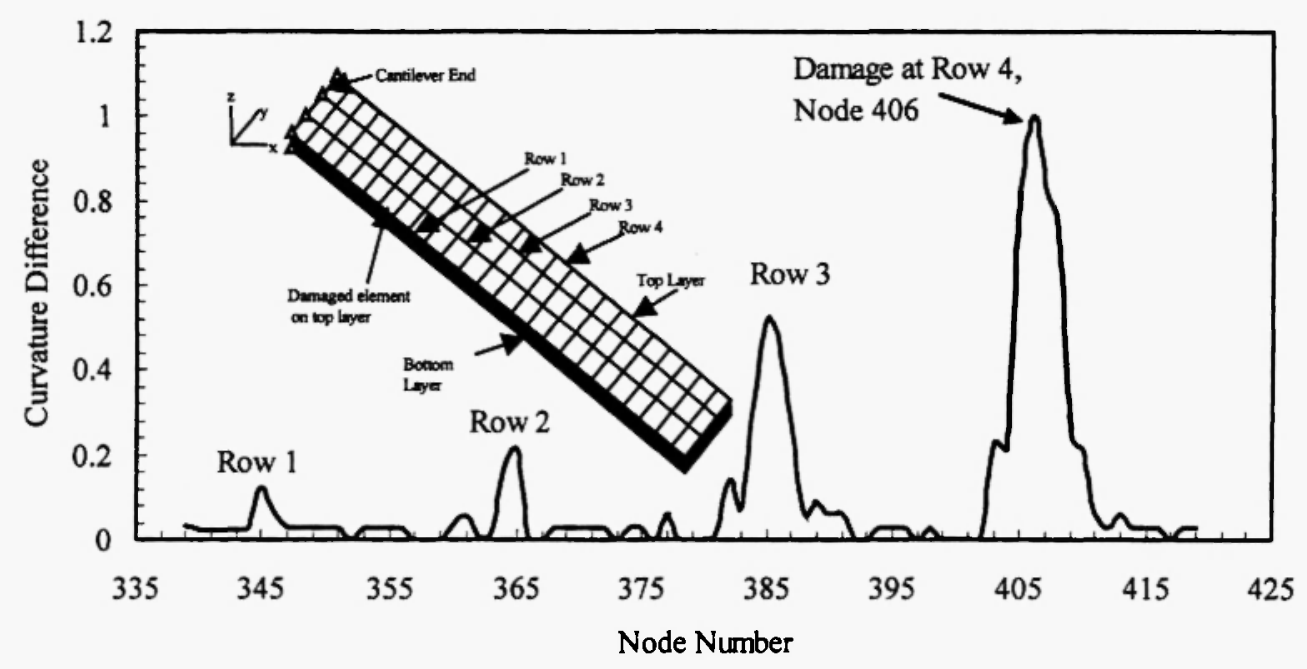

a. XY Plane

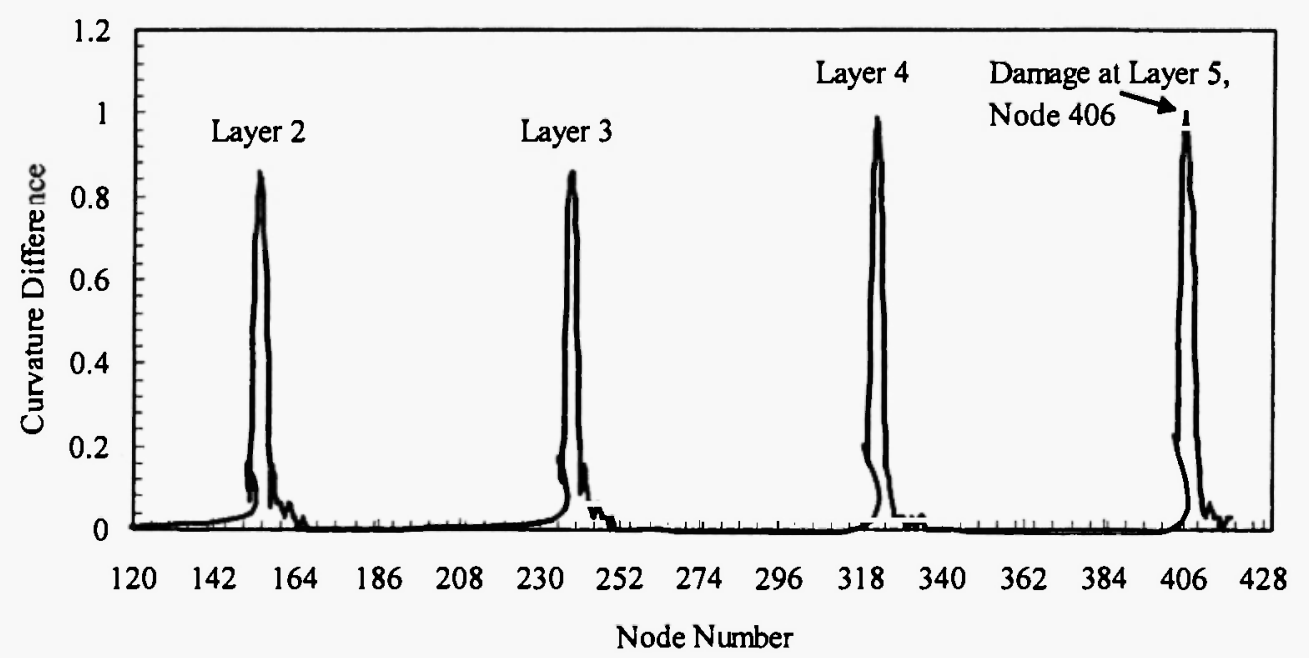

b. XZ Plane

Fig. 2: FEA model of laminated composite beam and corresponding elemental damage in the $\mathrm{XY}$ and $\mathrm{XZ}$ planes. 
the spikes - this presents a limitation of the technique. However, with careful observation, damage in most cases can still be identified in through-the-thickness direction, as shown in Fig. $2 b$ (node 406, on the top layer) for the model shown in Fig. 2a (inset). Nevertheless, a simultaneous analysis of XZ and XY is required (in the absence of detailed a priori knowledge of node numbering) for accurate damage site detection in 3D space.

Next, the procedure was applied to locate damage in two lamina facesheets and soft core sandwich composite beam shown in Fig. 3a(inset). The location of damage site within the core is distinctly obtained in 3D space ( $X Y$ and $X Z$ planes, respectively), as shown in Figs. 3a,b. In contrast, Fig. 4a (XY plane) clearly

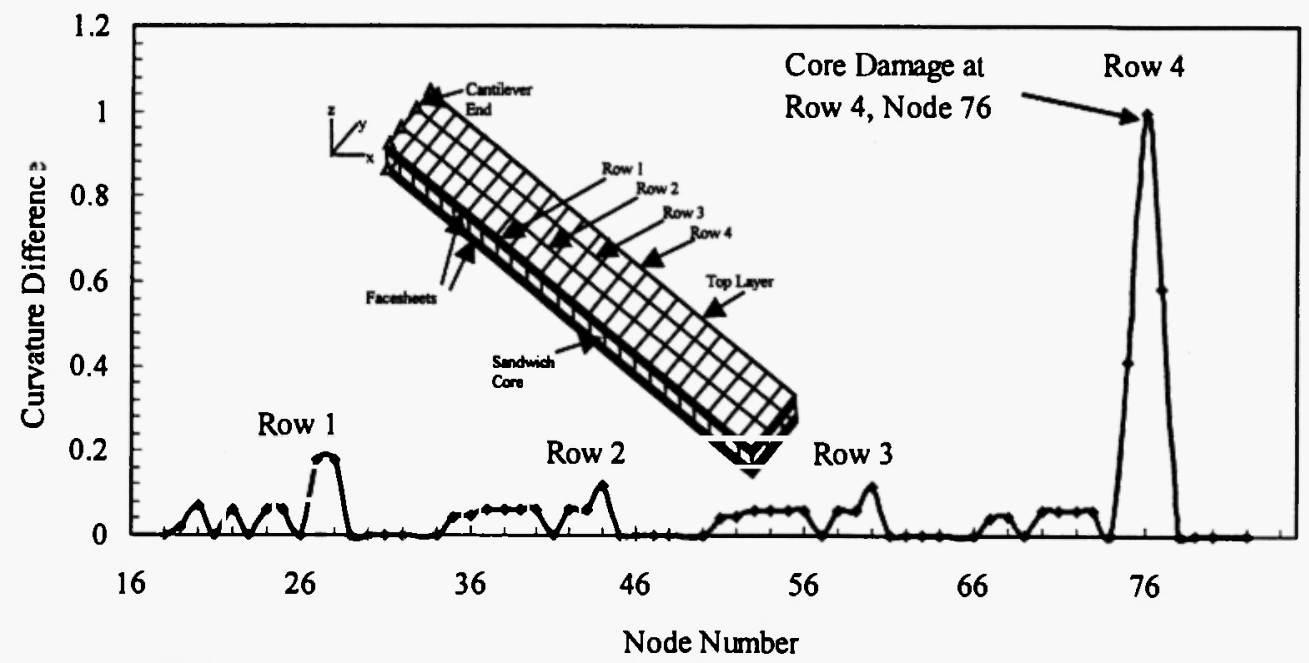

\section{a. XY Plane}

Node Number

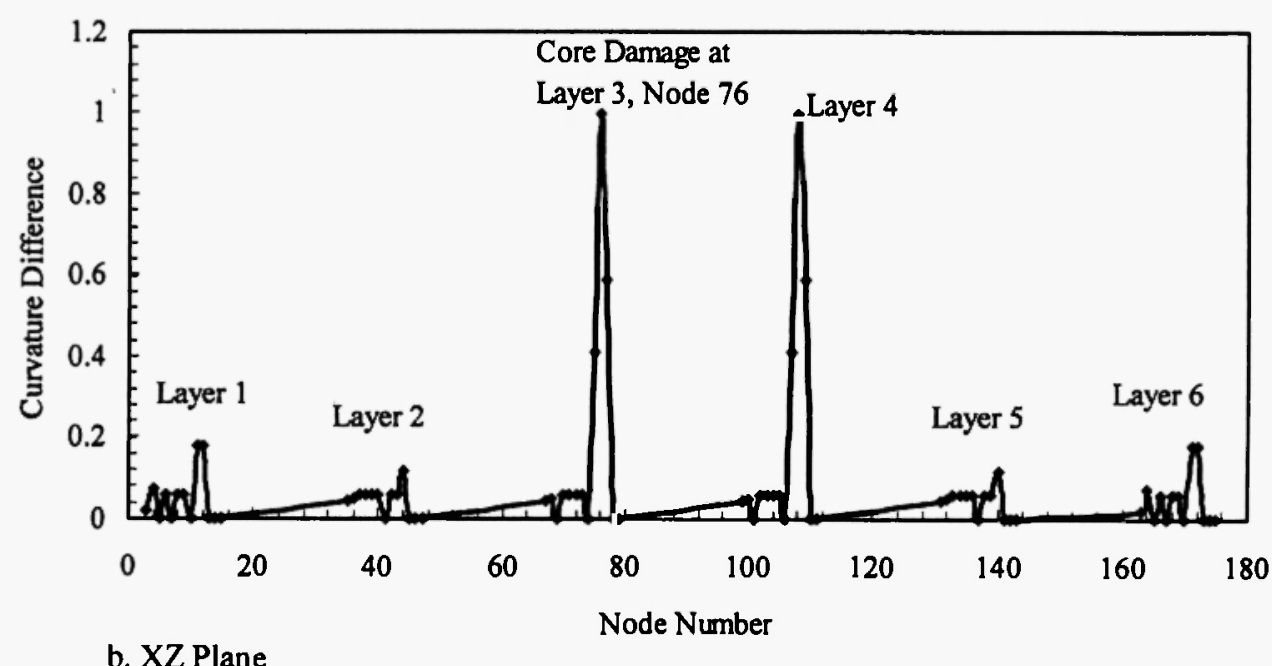

Fig. 3: FEA model of a typical sandwich composite beam and corresponding damage identification in the $\mathrm{XY}$ and $\mathrm{XZ}$ plane of the core. 
points to the damage being located on Row 4, however, through the thickness (XZ plane) analysis shown in Fig. $4 b$ yields peaks in each layer of almost identical magnitude, similar to the laminated case (Fig. $2 \mathrm{~b}$ ). Thus, it is difficult to point to damage site in through the thickness direction with any degree of accuracy using Fig. 4b. Facesheets are the primary load carrying constituents of the sandwich composite. Core, being significantly softer cannot resist the deformation caused by the reduced stiffness (damage) of the facesheets.

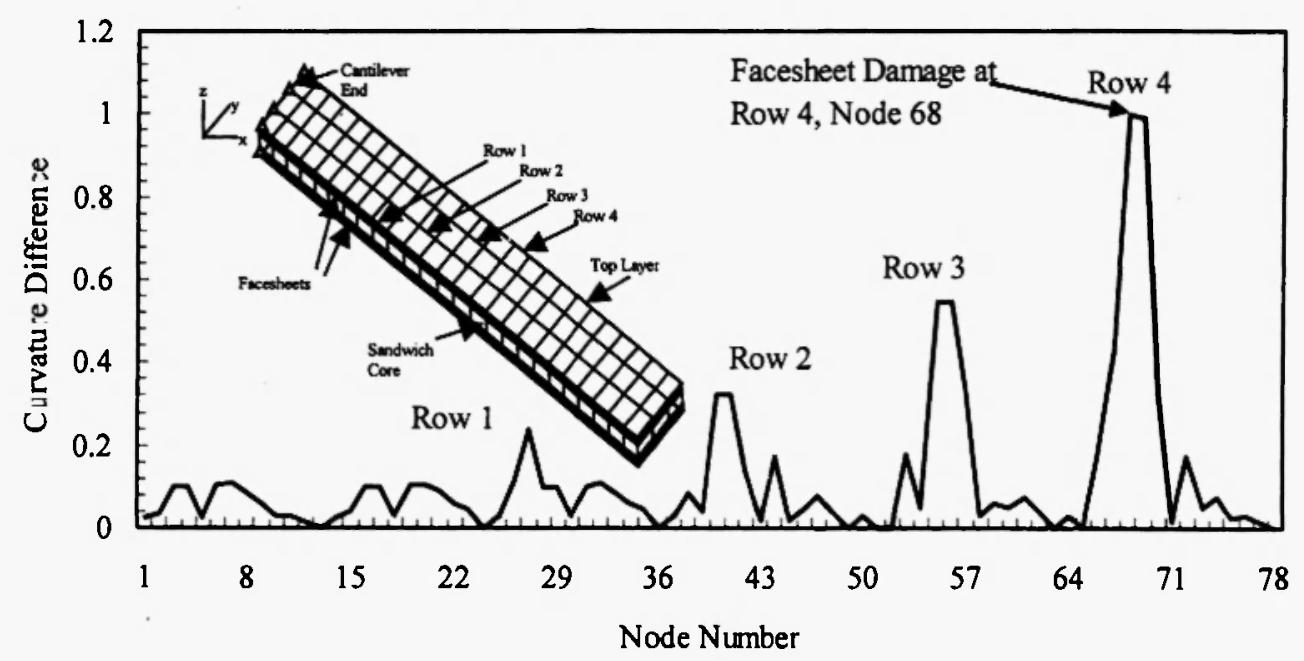

a. XY Plane

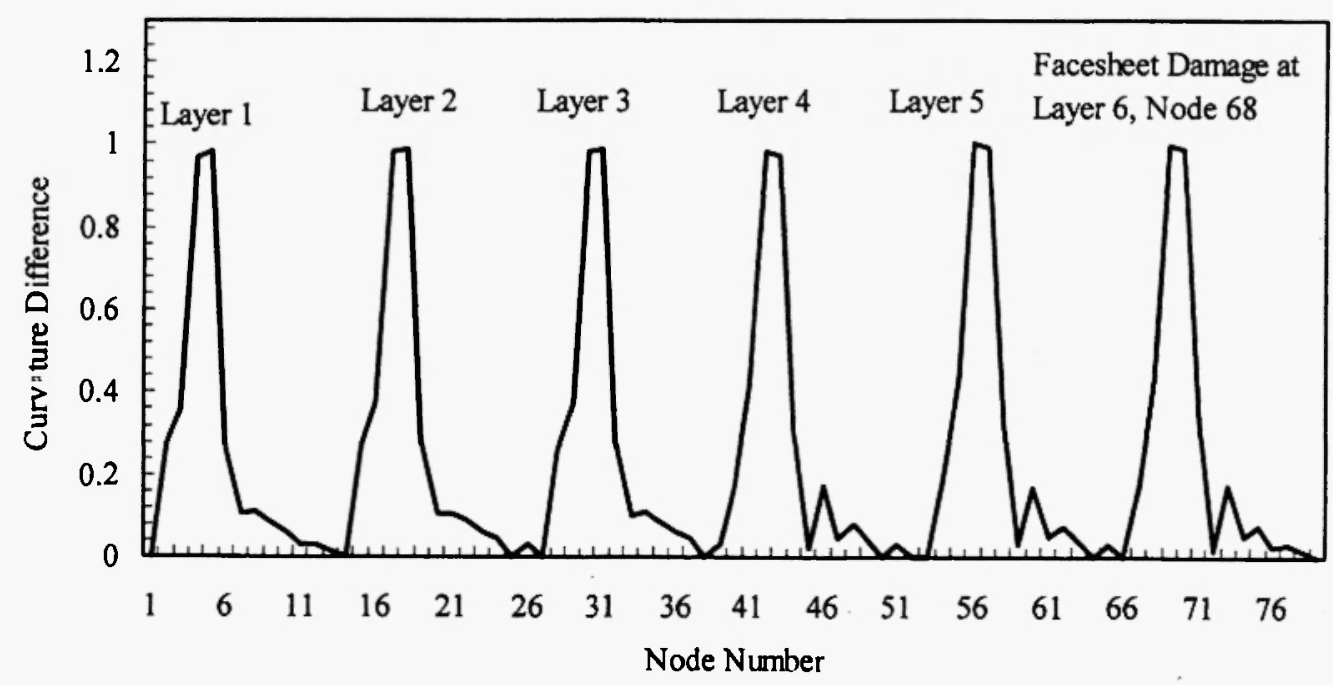

b. XZ Plane

Fig. 4: FEA model of a typical sandwich composite beam and corresponding damage identification in the $\mathrm{XY}$ and $\mathrm{XZ}$ plane of the facesheets. 
Therefore, facesheets displacement behavior dominates the global curvatures of individual constituents of the sandwich composite, thus the results shown in Fig. $4 \mathrm{~b}$. On the other hand, the damage in the low stiffness core is confined to the core and induces very little impact on the surrounding high stiffness facesheets. Hence, local damage in the soft core is more pronounced in both $\mathrm{XY}$ and $\mathrm{XZ}$ planes.

Finally, various sandwich composite ship hull structures were analyzed. Fig. 5a (inset) shows a representative ship hull model constructed with two high stiffness facesheets and a soft core. Core crushing and facesheet failure generally encountered under impact loading was the main focus of the study. Modeling

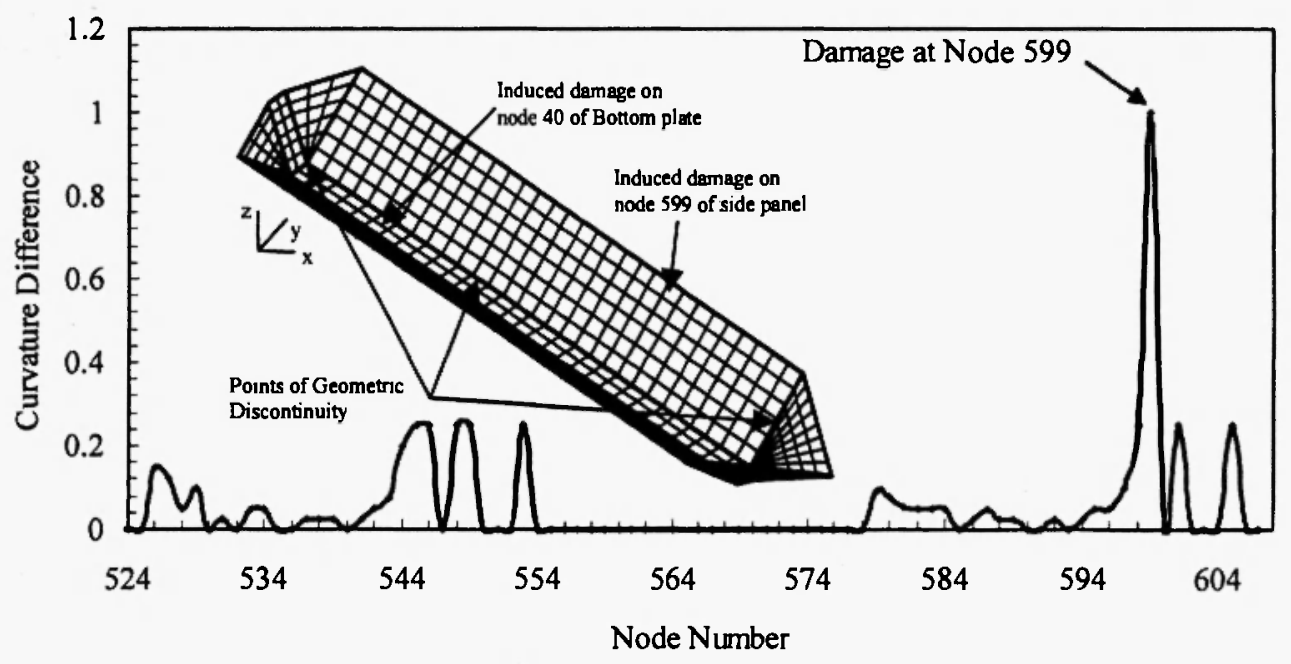

a. Side Panel Core

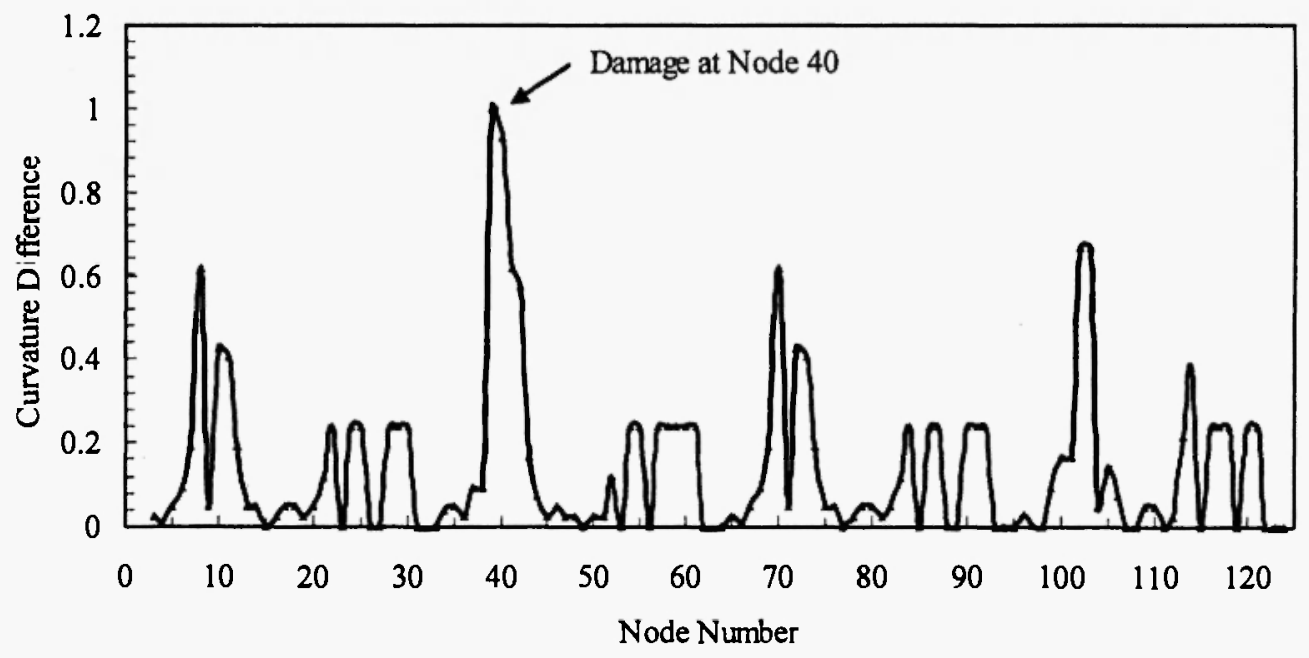

\section{b. Hull Bottom Facesheet}

Fig. 5: FEA model of a typical ship hull. Damage location in the core of the side panel and hull bottom facesheet identified. 
of multiple damage scenarios was necessary to simulate real life situations. In ship hull structures, special attention was paid to the node numbering in order to relate the spikes in the curve with the damage sites. Once again analysis was performed in both $\mathrm{XY}$ and $\mathrm{XZ}$ planes. Figs. 5a and 5b present typical (XY plane) results of damage at the side panel core and bottom plate facesheet of the ship hull, respectively. Damage is clearly identified by the spikes in the curve. In ship hulls, where the geometry is more complex, the density of the mesh was found to be a critical aspect of the analysis. For example, in the model shown in Fig. 5a (inset), 80 elements per section yielded substantial noise in the data that made the location of the damage inaccurate. However, when the number of elements on the section were increased by $60 \%$, a clear indication of the damage locations was obtained, which also aided in PNN convergence. An analysis performed to verify the response from the damage on the adjacent nodes in ship hulls yielded distinct peaks identifying the actual locations of the damage sites, similar to the behavior observed for isotropic case shown in Fig. 1 .

It was found that in 3D structures, displacement curvature analysis could not consider data at points of discontinuities. In order to obtain curvature, points must be planar and adjacent. Corner points, such as presented in Fig. 6 (inset), yielded false peaks that could be mistaken for damage locations. These false peaks are a result of the way curvature is calculated based on Eq. 5. Node 94 of Fig. 6, for example, can be easily mistaken for a damage site, but in reality it represents a point of discontinuity as do nodes 33 and 62 . In some cases multiple peaks can appear close to the damage site as a result of false curvatures, e.g., around node 128 of Fig. 6. This behavior turned out to be a problem not only for the physical interpretation of the results but also for the PNN training. To overcome this drawback, geometrically complex structures were limited to a segment-wise planar analysis; i.e., excluding the points of geometric discontinuities. Such difficulties were never encountercd in the literature, as only simple cantilever beams were analyzed in most cases $/ 7-9 /$. In

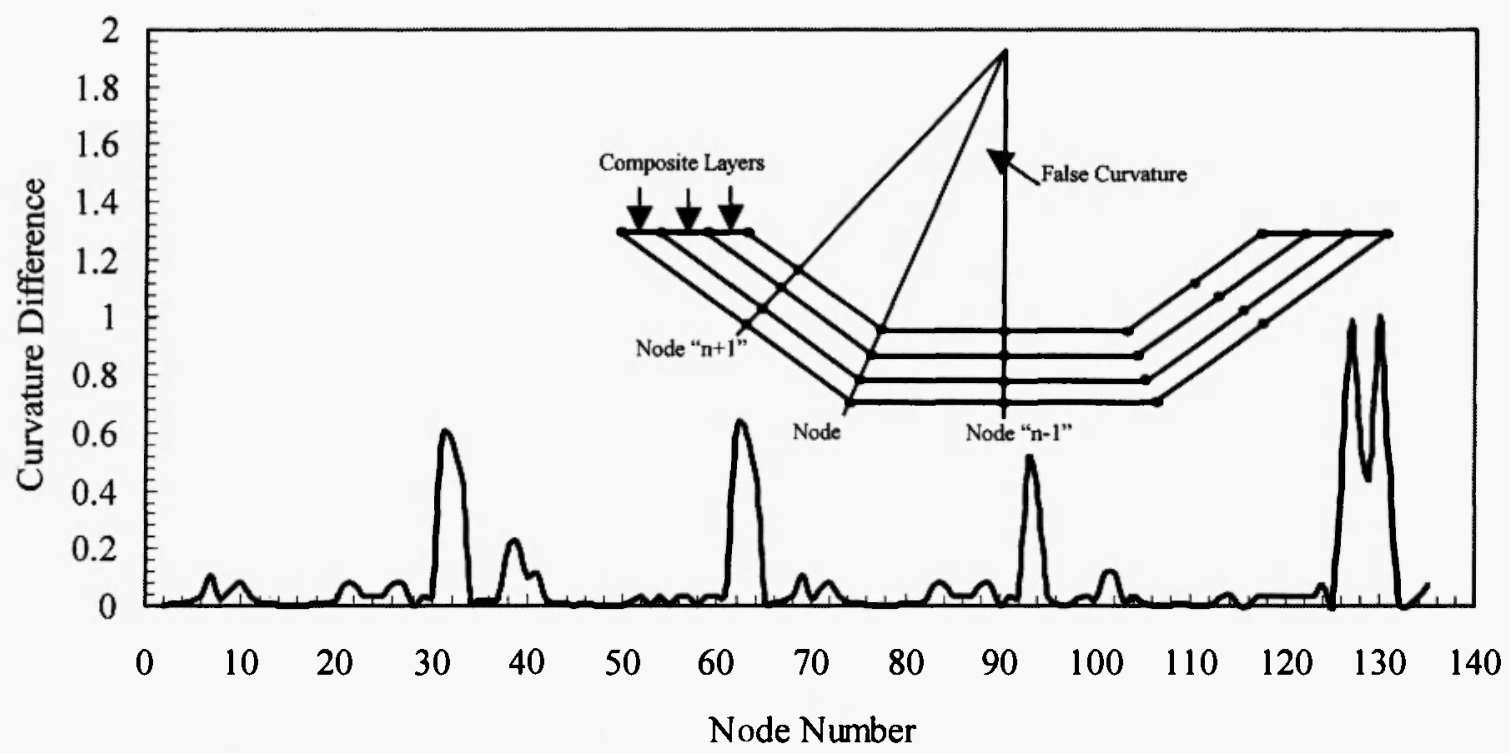

Fig. 6: Curvature calculation for edge nodes and corresponding false peaks. 


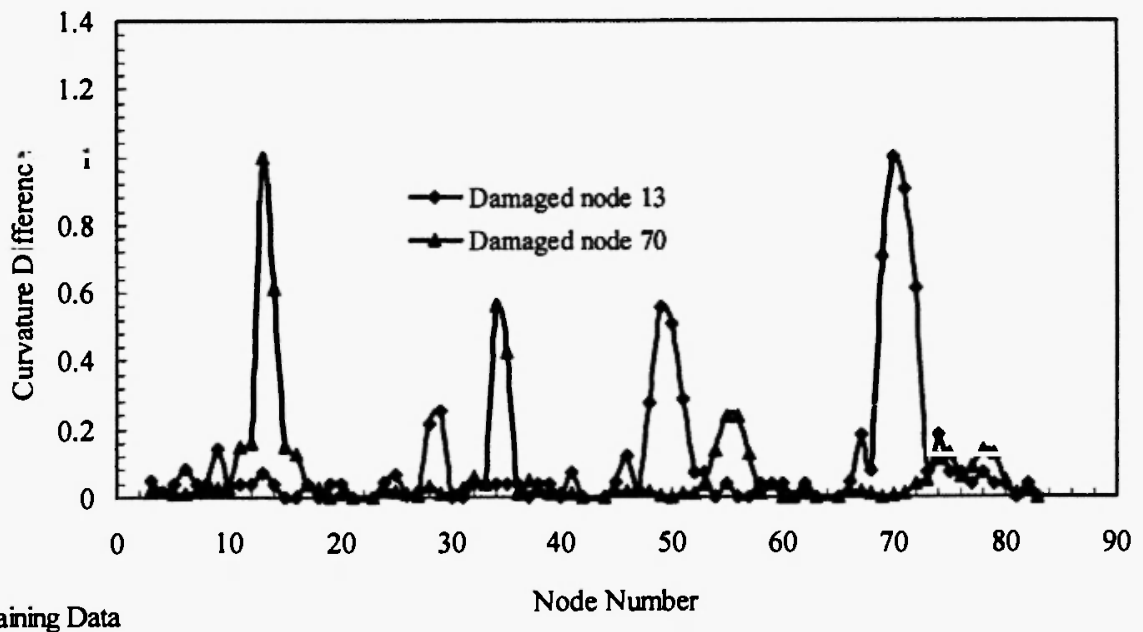

a. Training Data

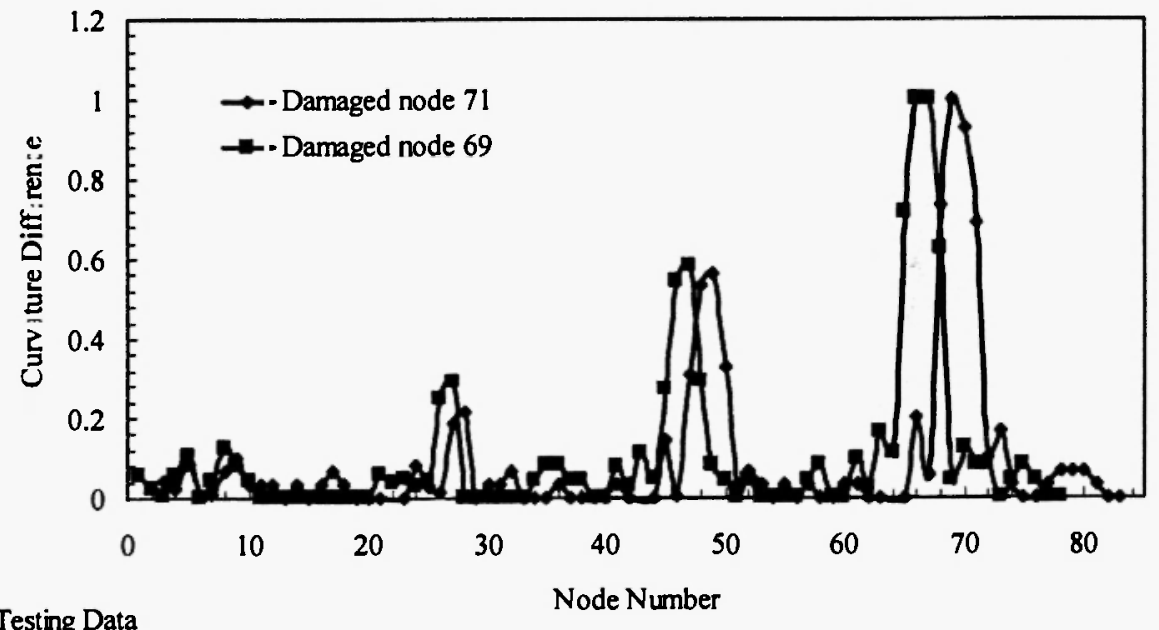

b. Testing Data

Node Number

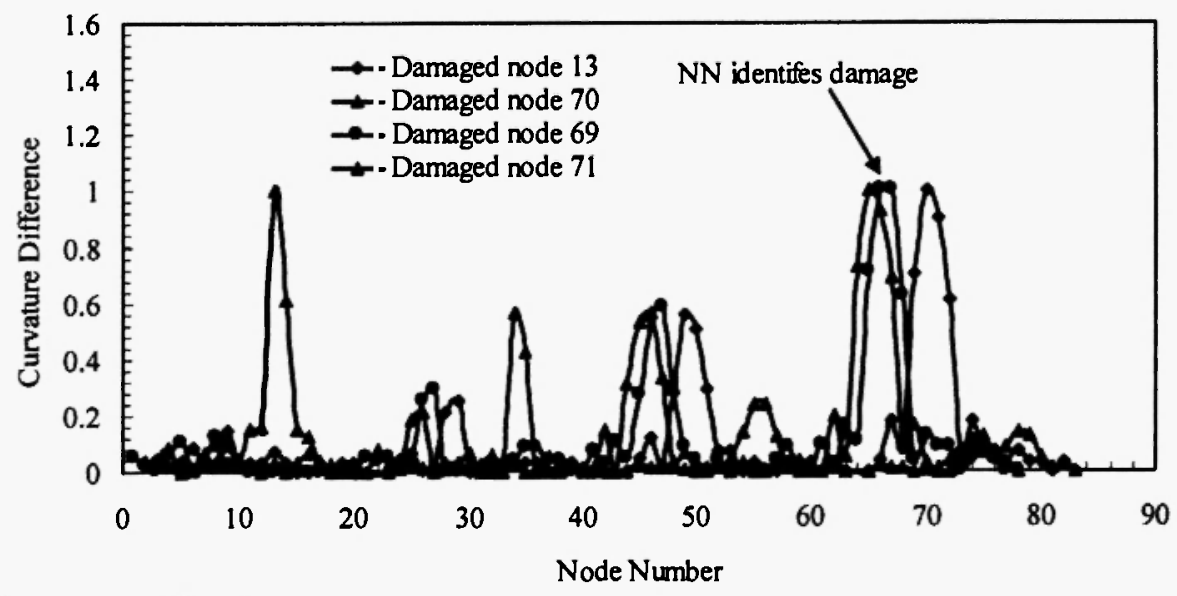

c. Convergence of Results

Fig. 7: PNN training, testing and convergence curves identifying the exact location of damage. 
theory, curved members can be analyzed using the displacement curvature technique by reformulating Eq. 5 to account lor the initial geometry and curvature.

A PNN was subsequently trained to detect damage in sandwich composites and ship hull structures. Fig. 7a shows two damage sites in the curve of a typical beam model that were used to train the PNN. Whereas, Fig. $7 \mathrm{~b}$ shows the PNN testing data to see how well it works. It can be seen that each damage location has a specific curve that is completely different and is thus representative of individual damage location. Fig. 7c shows the convergence behavior of PNN technique. In all cases, the PNN related the test plots with the learned bchavior plots and successfully pointed to the damage site with remarkable accuracy. For displacement curvature cases that did not resemble learning curves, PNN output a curve that most resembled what it had learned. PNN trained with FEA data from simple beam models and tested to simulate damage in complex ship hull structures provided very encouraging results in segmental planar analysis.

\section{CONCLUSIONS}

This paper extends the displacement curvature damage detection idea to complex 3D orthotropic sandwich composite structures. The proposed method utilized direct difference between the fundamental mode of undamaged and damaged structure and clearly identified damage location as a spike in the curve in planar isotropic and orthotropic structures. However, points of geometric discontinuitics yielded inaccurate results and subsequently confused the PNN to recognize the damage sites. To overcome this problem, a segment-wise planar analysis is proposed that successfully identified damage in various constituents of a complex sandwich ship hull structure. In general, 3D mesh refinement and node numbering demanded substantial effort.

\section{REFERENCES}

1. M. Burnam, Fatigue Behavior Initiation and Propagation in Sandwich Structures, Royal Institute of Technology, Report No. 98-29, ISSN 0280-4646, Sweden.

2. D. Zenkert: The Handbook of Sandwich Construction, Chameleon Press, London, 1997.

3. Y. Yu: Vibrations of Elastic Plates, Springer, New York, 1995.

4. P. Cawley and R. Adams: The location of defects in structures from measurements of natural frequencies, Journal of Strain Analysis, 14 (2), $49-57$ (1979).

5. M. Yuen: A numerical study of the eigenparameters of a damaged cantilever, Journal of Sound and Vibration, 103 (3), 301-310 (1985).

6. D. Pilkcy, Computation of a Damping Matrix for Finite Element Updating, Dissertation, VPI\&SU, April 1998. 
7. A. Pandey, M. Biswas and M. Samman: Damage detection from changes in curvature mode shapes, Journal of Sound and Vibration, 145 (2), 321 -332 (1991).

8. C. Ratcliffe and W. Bagaria: Vibration technique for locating delamination in a composite beam, AIAA Journal, 36 (6), 1071-1077 (1998).

9. C. Ratcliffe: Damage detection using a modified Laplacian operator on mode shape data, Journal of Sound and Vibration, 204 (3), 505-517 (1997).

10. J. Mackerle: Finite element analysis of sandwich structures: A bibliography (1980-2001), Engineering Computations, 19 (2), 206-245 (2002).

11. J. Reddy: Energy and Variational Methods in Applied Mechanics, John Wiley, 1984.

12. P. Wasserman: Advanced Methods in Neural Networks, Von Nostrans Reinhold, New York, 1993.

13. O. Byon and Y. Nishi: Damage identification of CFRP laminated cantilever beam by using neural network, Key Engineering Materials, 141-143, 55-64 (1998).

14. T. Marwala: Damage identification using committee of neural networks, Journal of Engineering Mechanics, 126 (1), 43-50 (2000).

15. T. Marwala: Probabilistic fault identification using a committee of neural networks and vibration data, AIAA Journal of Aircraft, 38 (1), 65-73 (2001). 
